\title{
COMPARACIÓN ENTRE MÉTODOS DE CORRECCIÓN DE SESGOS PARA LAS PROYECCIONES DE CAMBIO CLIMÁTICO EN LOS PIRINEOS
}

\author{
Amblar-Francés P. ${ }^{(1)}$, Ramos-Calzado P. ${ }^{(2)}$, Pastor Saavedra A. ${ }^{(3)}$, \\ Rodríguez Camino E. ${ }^{(4)}$ \\ (1), (2) AEMET Avda. Américo Vespucio, 3, 41092, Sevilla. \\ mamblarf@aemet.es, pramosc@aemet.es \\ (3), (4) AEMET Calle Leonardo Prieto Castro, 8, 28071, Madrid. \\ mpastors@aemet.es, erodriguezc@aemet.es
}

\section{Resumen}

Las distribuciones de las variables climáticas procedentes tanto de los GCM como de las técnicas de regionalización pueden diferir de las distribuciones observadas de dichas variables. Estas diferencias pueden ser importantes y pueden afectar a determinados índices climáticos utilizados por la comunidad de impactos. Para minimizar este efecto se utilizan técnicas dirigidas a corregir estas diferencias, los métodos de corrección de sesgo. En este trabajo se han analizado tres métodos de corrección de sesgo: dos basados en el denominado empirical quantile mapping y otro basado en la varianza. Estos métodos se han aplicado a las proyecciones de temperaturas extremas (temperatura máxima y mínima) de 9 estaciones distribuidas por una zona montañosa, la zona pirenaica, las cuales son especialmente sensibles a los efectos del cambio climático.

Palabras claves: corrección de sesgos, quantile mapping, cambio climático, Pirineos

\section{Introducción}

Este trabajo se desarrolla en el marco del proyecto de "Caracterización de la evolución del clima y provisión de información para la adaptación en los Pirineos (CLIMPY)", siendo uno de sus objetivos el conocimiento de la evolución del clima en esta zona tan característica bajo diferentes escenarios futuros. En el quinto informe (IPCC, 2013) del Panel Intergubernamental del Cambio Climático (IPCC) se identificaron las zonas de montaña como espacios particularmente sensibles al cambio climático. En concreto, los Pirineos son un espacio geográfico singular puesto que presenta una amplia diversidad climática desde el clima oceánico hasta el clima mediterráneo, pasando por zonas de clima con una tendencia más continental. Sin embargo, esta singularidad y la propia variabilidad presente no siempre quedan representadas en las proyecciones futuras obtenidas por los modelos climáticos globales (en sus siglas en inglés, GCMs), siendo necesario el uso de técnicas de regionalización. Sin embargo, estos datos regionalizados o bajados de escala presentan errores sistemáticos que son debidos a la discretización y a los promedios utilizados en las celdas (Teutschbein and Seibert, 2012), por ello, es importante aplicar una corrección de los datos mediante métodos que permitan reducir el sesgo en sus estadísticos.

\section{Área de estudio y datos}

Las zonas de montaña, con sus fuertes gradientes ambientales asociados, se encuentran entre las áreas más sensibles y vulnerables al cambio climático. Este trabajo se focaliza 
en la zona de los Pirineos, que es el macizo montañoso situado entre España, Andorra y Francia. Los Pirineos se extienden desde el mar Mediterráneo hasta el océano Atlántico y presenta zonas montañosas con picos superiores a 3.000 metros, pequeños glaciares, lagos, valles y cañones. Este espacio geográfico presenta una diversidad climática que se extiende desde el clima oceánico hasta el clima mediterráneo, pasando por zonas de clima con una tendencia más continental. Se parte de un conjunto de 338 estaciones con dato diario de temperatura máxima y mínima en la región de los Pirineos. Estas estaciones han sido facilitadas por las instituciones socias del proyecto CLIMPY (AEMET, Météo-France, CENMA y MeteoCat), y se han seleccionado 9 en base a que cumplan condiciones de homogeneidad y que el número de datos ausentes sea inferior al $2 \%$ en el periodo 1961-2000. Esta selección incluye estaciones tanto costeras como de montaña (ver Fig.1).

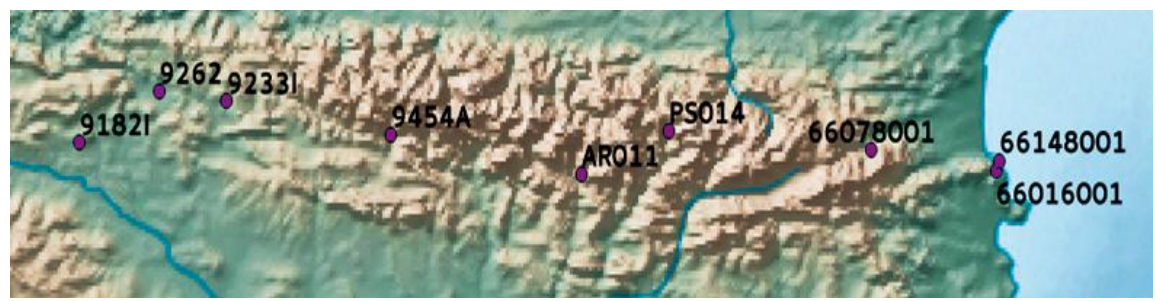

Fig. 1. - Estaciones seleccionadas para este estudio.

\begin{tabular}{|lccc|}
\hline MODEL0 & RCP 8.5 & RCP6.0 & RCP 4.5 \\
\hline ACCESS1.3 & $\mathrm{X}$ & & \\
\hline bcc-csm1-1-m & $\mathrm{X}$ & $\mathrm{X}$ & $\mathrm{X}$ \\
\hline BNU-ESM & $\mathrm{X}$ & & $\mathrm{X}$ \\
\hline CanESM2 & $\mathrm{X}$ & & $\mathrm{X}$ \\
\hline CMCC-CESM & $\mathrm{X}$ & & \\
\hline CMCC-CM & $\mathrm{X}$ & & $\mathrm{X}$ \\
\hline CMCC-CMS & $\mathrm{X}$ & & \\
\hline CSIRO-Mk3-6-0 & $\mathrm{X}$ & $\mathrm{X}$ & \\
\hline GFDL-ESM2G & $\mathrm{X}$ & & $\mathrm{X}$ \\
\hline inmcm 4 & $\mathrm{X}$ & & $\mathrm{X}$ \\
\hline IPSL-CM5A-LR & $\mathrm{X}$ & $\mathrm{X}$ & $\mathrm{X}$ \\
\hline IPSL-CM5A-MR & $\mathrm{X}$ & $\mathrm{X}$ & $\mathrm{X}$ \\
\hline IPSL-CM5B-LR & $\mathrm{X}$ & & $\mathrm{X}$ \\
\hline MIROC-ESM & $\mathrm{X}$ & & $\mathrm{X}$ \\
\hline MIROC-ESM-CHEM & $\mathrm{X}$ & $\mathrm{X}$ & $\mathrm{X}$ \\
\hline MIROC5 & $\mathrm{X}$ & $\mathrm{X}$ & $\mathrm{X}$ \\
\hline MPI-ESM-LR & $\mathrm{X}$ & & $\mathrm{X}$ \\
\hline MPI-ESM-MR & $\mathrm{X}$ & & $\mathrm{X}$ \\
\hline MRI-CGCM3 & $\mathrm{X}$ & $\mathrm{X}$ & $\mathrm{X}$ \\
\hline
\end{tabular}

Tabla 1.- Proyecciones de cambio climático utilizadas, indicando el GCM y RCP.
Además, se han utilizado las proyecciones de cambio climático de dichas estaciones calculadas a partir de una selección de GCM del proyecto de intercomparación de modelos CMIP5 (del inglés, Phase Five of the Coupled Model Intercomparison Project), utilizados también en el quinto informe (AR5) del IPCC (IPCC, 2013) y del método de regionalización estadística basado en regresión lineal (Brunet et al., 2009), (veáse tabla 1). Estas proyecciones se han obtenido bajo tres sendas representativas de concentración (RCP de su acrónimo en inglés): RCP4.5, RCP6.0 y RCP8.5. 


\section{Metodología}

Ante las diferencias existentes entre las distribuciones de probabilidad de las variables climáticas procedentes de las proyecciones de los GCM en el escenario historical frente a las de los valores observados, obtenidas a partir de la técnica de regionalización estadística basada en regresión lineal (como se observa en la Fig.2), surge la necesidad de corregir estas discrepancias (Piani et al., 2010).
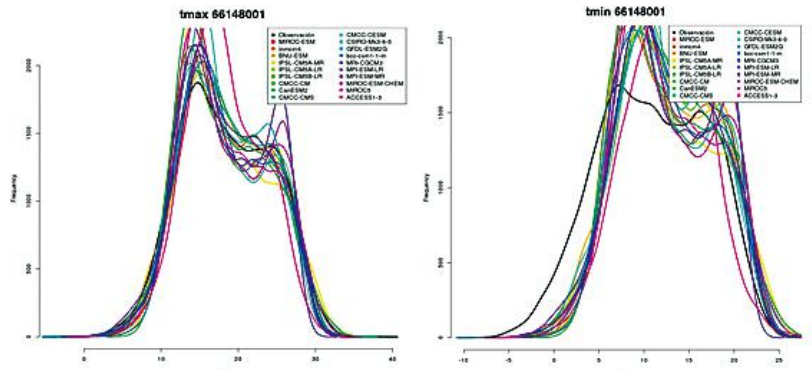

Fig. 2- Representación de la distribución de frecuencia observada (negro) y de las simuladas con los diferentes GCM de CMIP5 seleccionados para la temperatura máxima (izquierda) y mínima (derecha), en el periodo 1961-2000 y para la estación 66148001.

Para ello, se suelen utilizar métodos de corrección de sesgos cuyo objetivo es obtener unas proyecciones de cambio climático más realistas que recojan las características climáticas locales de la zona y que se puedan utilizar para realizar estudios de impacto y vulnerabilidad más adaptados a la realidad. En este trabajo, se ha realizado un análisis comparativo entre tres métodos de correcciones de sesgos: dos basados en el denominado empirical quantile mapping (eQM) (Amengual et al., 2012) y otro basado en la varianza (Piani et al., 2010; Chen et al., 2010) para las proyecciones de cambio climático de las temperaturas extremas (temperatura máxima y mínima).

El método $e Q M$ es un método basado en estudiar la distribución de los valores observados de la variable climática y realizar un ajuste de algunas de las características de la función de distribución de probabilidad empírica con los valores proyectados, para posteriormente aplicar el resultado de este ajuste a las proyecciones, tanto en el periodo de clima actual (1961-2000) como en el periodo 2006-2100; de forma que las simulaciones queden corregidas. Además, se ha introducido una variante de este método, $e Q M(n+1)$, basada en $e Q M$ donde se tiene en cuenta que se está trabajando con una muestra y no con toda la población, por ello en el cálculo de los percentiles se ha cambiado el denominador " $n$ " por " $n+1$ ", siendo " $n$ " el tamaño de la muestra utilizada (Coles, 2001). Por otro lado, el tercer método de corrección analizado, el método de la varianza trata de corregir la media y la varianza de las series temporales de temperatura y es aplicable solo a la temperatura (Chen et al., 2010).

\section{Resultados}

Dependiendo de la estación y de la variable de que se trate, se encuentran o bien diferencias en la forma de la distribución, o bien un desplazamiento de los valores. Por tanto, las proyecciones pueden subestimar o sobrestimar las variables, tal y como se observa en la Fig.3, en la cual se comparan las distribuciones de probabilidad empírica acumulada 
(ECDF) de los valores observados frente a los simulados con y sin corregir por los métodos: (i) eQM, (ii) eQM(n+1) y (iii) varianza para las temperaturas máxima y mínima en el periodo comparativo 1961-2000 para una estación.
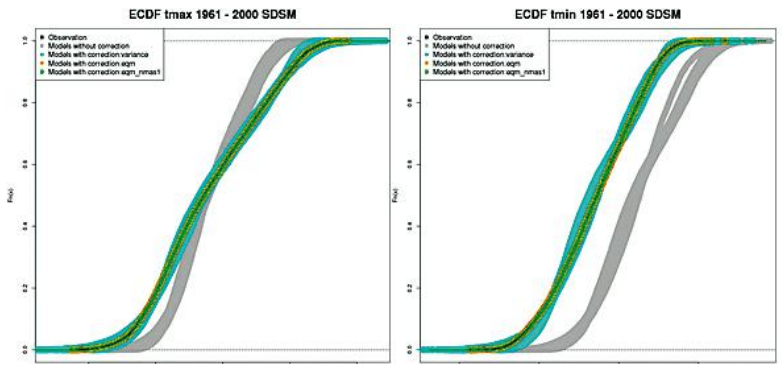

Fig. 3- Representación de la ECDF de los valores observados (negro) y de las simuladas sin corregir (gris) y corregidas por los métodos: (i) eQM (naranja), (ii) eQM(n+1) (verde) y (iii) varianza (azul) para la temperatura máxima (izquierda) y mínima (derecha) en el periodo 1961-2000 para la estación 66148001.

En ambos casos, las distribuciones de valores obtenidos con los dos primeros métodos (zonas naranja y verde) se adaptan más a la obtenida a partir de los datos observacionales (línea negra) que las obtenidas con el método de la varianza (zona azul).

Cuando las proyecciones futuras de temperatura máxima y mínima se corrigen con los métodos indicados y se calculan los valores medios anuales para los escenarios futuros utilizados se obtienen resultados similares entre las correcciones, tal y como se puede observar en la Fig. 4.
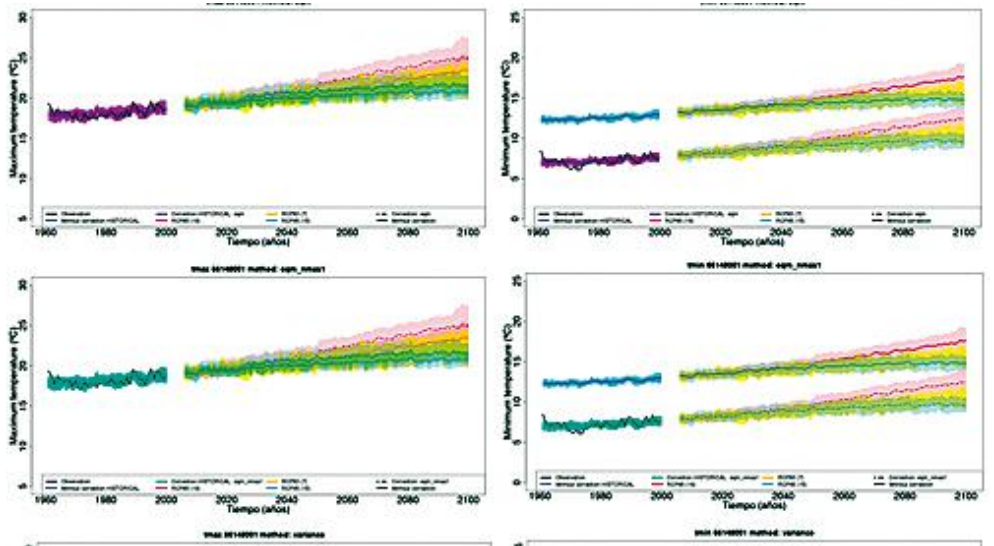

Fig. 4-

Evolución del valor medio anual de la temperatura máxima (izquierda) y mínima (derecha) corregida con los tres métodos empleados en los periodos 1961-2000 y
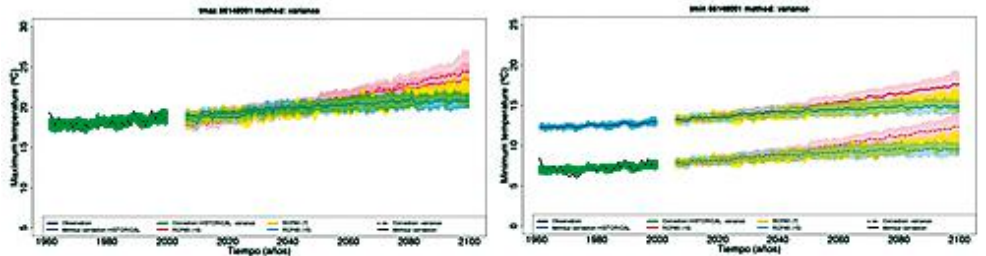
2006-2100: (i) eQM, (ii) eQM $(n+1)$ y (iii) varianza para la estación $66148001 \mathrm{y}$ bajo los tres escenarios. 
Analizando sus anomalías y la de sus índices extremos: número de días cálidos, longitud de la ola de calor, número de días de helada y número de noches cálidas (AmblarFrancés P. et al, 2017), se obtienen pocas diferencias entre sus valores corregidos y sin corregir. Como se observa en la Fig.5 para el caso de la temperatura máxima y los índices extremos asociados a ella, las evoluciones son muy similares.
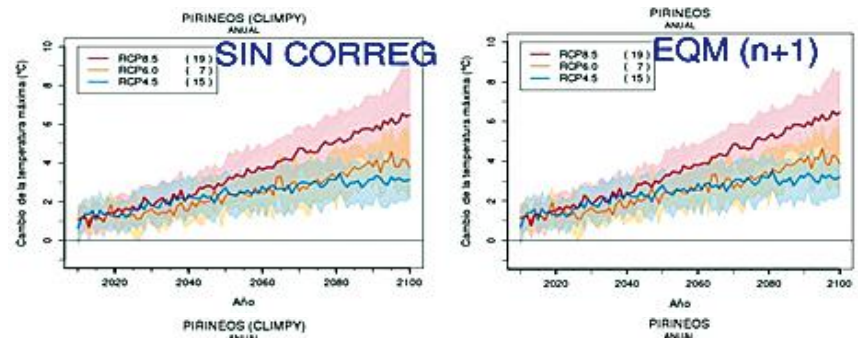

Fig.5- Evolución de la anomalía media anual proyectada sin
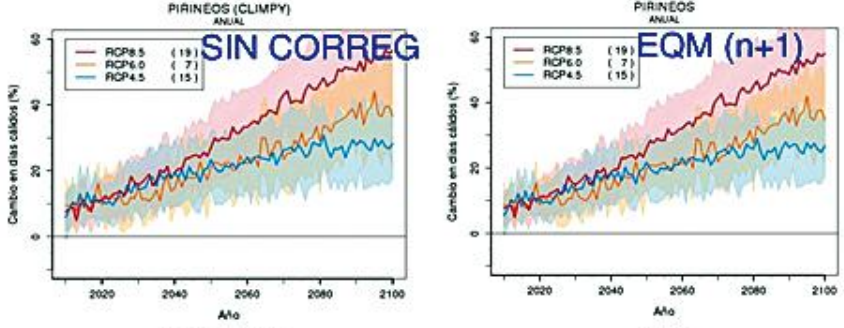
corregir (izquierda) y corregida con el método (iii) $\mathrm{eQM}(\mathbf{n}+1)($ derecha $)$ de la temperatura máxima (panel superior), el número de días cálidos (panel medio) y la longitud de la ola de calor
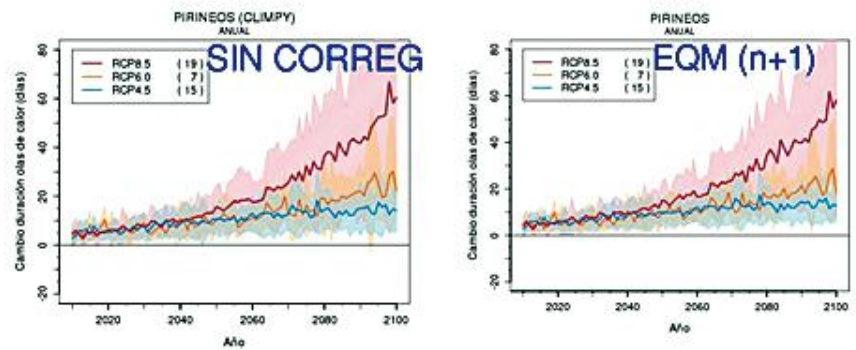
(panel inferior) para la región pirenaica en el periodo 2006-2100.

\section{Conclusiones}

Se han aplicado tres métodos de corrección de sesgo (varianza, eQM y eQM(n+1)) a los valores proyectados de temperatura máxima y mínima de las diferentes estaciones seleccionadas de la zona pirenaica y se observa que los tres métodos mejoran sustancialmente las proyecciones en el periodo de calibración. No obstante, los métodos basados en la función de probabilidad empírica acumulada reproducen de manera más fidedigna los valores observados. Para el tratamiento de la temperatura máxima y mínima no se aprecian diferencias notables entre estos, salvo en las colas de la distribución. Por ello, nos decantaríamos por el método que hemos denominado $e \mathrm{QM}(n+1)$ al reproducir mejor los valores extremos. 
Tal y como cabría esperar, las correcciones de sesgo cobran importancia al tratar los valores absolutos de las variables, en cambio, cuando se estudia la anomalía no se observan cambios relevantes ni en la tendencia ni en los valores durante el periodo de proyección futuro a excepción del periodo final, en el que se aprecian valores menores en las proyecciones corregidas que en las que están sin corregir, así como una ligera disminución en la dispersión entre modelos.

\section{Referencias}

- Amblar-Francés P., Casado-Calle, M. J., Pastor-Saavedra, M. A., Ramos-Calzado P., RodríguezCamino, E., 2017. Guía de escenarios regionalizados de cambio climático sobre España a partir de los resultados del IPCC-AR5. Ministerio de Agricultura y Pesca, Alimentación y Medio; Agencia Estatal de Meteorología: 96 pp., Madrid

- Amengual, A., Homar V., Romero R., Alonso S., and Ramis C., 2012. A Statistical Adjustment of Regional Climate Model Outputs to Local Scales: Application to Platja de Palma, Spain. J. Climate, 25, 939-957. doi: http://dx.doi.org/10.1175/JCLI-D-10-05024.1

- Brunet, M., Casado, M.J, de Castro, M., Galán, P., López, J. A., Martín, J.M., Pastor, A., Petisco, E., Ramos, P., Ribalaygua, J., Rodríguez, E., Sanz, I. \& Torres, L., 2009. Generación de escenarios regionalizados de cambio climático para España. Ministerio de Medio Ambiente y Medio Rural y Marino; Agencia Estatal de Meteorología: 158 pp., Madrid

- Chen J., Brissette F. P., Poulin A., and Leconte R., 2011, Overall uncertainty of the hydrological impacts of climate change for a Canadian watershed, Water Resour. Res., 47, W12509, doi:10.1029/ 2011WR010602.

- Coles, S.,2001. An introduction to statistical modeling of extreme values. Springer Series in Statistics. Springer Verlag London. 208 pp.

- IPCC, 2013: Climate Change 2013: The Physical Science Basis. Contribution of Working Group I to the Fifth Assessment Report of the Intergovernmental Panel on Climate Change [Stocker, T.F., D. Qin, G.-K. Plattner, M. Tignor, S.K. Allen, J. Boschung, A. Nauels, Y. Xia, V. Bex and P.M. Midgley (eds.)]. Cambridge University Press, Cambridge, United Kingdom and New York, NY, USA, 1535 pp. Available:http://www.ipcc.ch/report/ar5/ 2.

- Piani, C., Weedon, G. P., Best, M., Gomes, S. M., Viterbo, P., Hagemann, S., \& Haerter, J. O., 2010, Statistical bias correction of global simulated daily precipitation and temperature for the application of hydrological models. Journal of Hydrology, 395, 199-215. doi:10.1016/j.jhydrol.2010.10.024

- Teutschbein, C. and Seibert J., 2012, Bias correction of regional climate model simulations for hydrological climate-change impact studies: Review and evaluation of different methods. J Hydrol 456-457, pp 12-29, doi:10.1016/j.jhydrol.2012.05.052. 